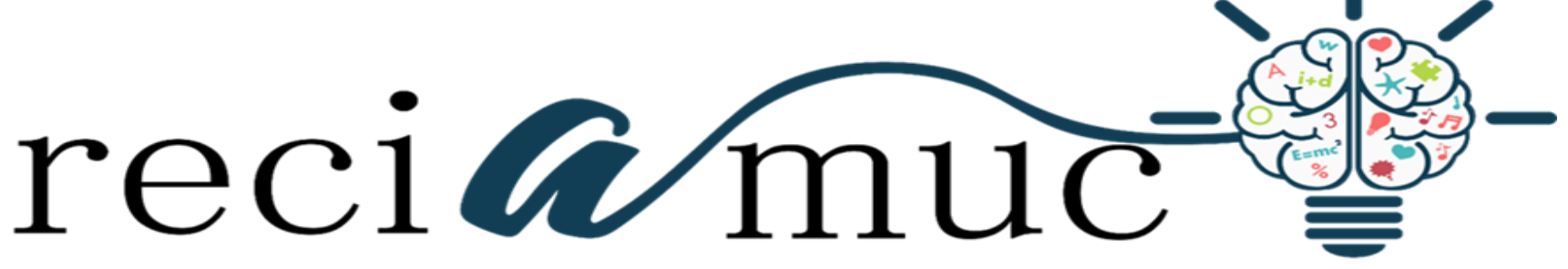

Revista científica de investigación actualización del mundo de las ciencias

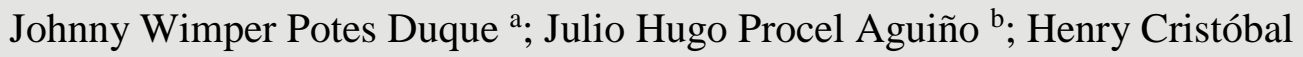 \\ Yoncón Ramírez ${ }^{\text {c }}$ Alexandra Asunción Vanegas Vélez ${ }^{\text {d }}$
}

Factores de pronósticos en embarazo tras la inseminación intrauterina

Factors of forecasts in pregnancy after intrauterine insemination

Revista Científica de Investigación actualización del mundo de las Ciencias. Vol. 3

núm., 3, julio, ISSN: 2588-0748, 2018, pp. 544-567

DOI: $10.26820 /$ reciamuc/3.(3).julio.2019.544-567

URL: http://reciamuc.com/index.php/RECIAMUC/article/view/303

Código UNESCO: 3201.08 Ginecología

Tipo de Investigación: Artículo de Revisión

(C) RECIAMUC; Editorial Saberes del Conocimiento, 2019

Recibido: 01/06/2019 Aceptado: 19/06/2019 Publicado: 01/07/2019

Correspondencia: johnnypotes_62@hotmail.com

a. Magister en Gerencia en Salud para el Desarrollo Local; Magister en Salud Publica; Especialista en Gerencia y Planificación Estratégica de Salud; Especialista en Ginecología y Obstetricia; Diploma Superior en Desarrollo Local y Salud; Doctor en Medicina y Cirugía; Universidad de Guayaquil; Guayaquil, Ecuador; johnnypotes 62@ hotmail.com

b. Magister en Epidemiologia; Especialista en Ginecología y Obstetricia; Diplomado en Docencia Superior; Doctor en Medicina y Cirugía; Universidad de Guayaquil; Guayaquil, Ecuador.

c. Magister en Salud Publica; Diploma Superior en Epidemiologia; Medico; Universidad de Guayaquil; Guayaquil, Ecuador.

d. Magister en Gerencia en Salud para el Desarrollo Local; Especialista en Gerencia y Planificación Estratégica en Salud; Diploma Superior en Desarrollo Local y Salud; Especialista en Ginecología y Obstetricia; Doctora en Medicina y Cirugía; Universidad de Guayaquil; Guayaquil, Ecuador. 


\title{
Factores de pronósticos en embarazo tras la inseminación intrauterina
}

Vol. 3, núm. 3., (2019)

Johnny Wimper Potes Duque; Julio Hugo Procel Aguiño; Henry Cristóbal Yoncón Ramírez;

Alexandra Asunción Vanegas Vélez

\section{RESUMEN}

La inseminación intrauterina, es un proceso caracterizado por depositar un grupo de espermatozoides previamente seleccionados y preparados en el conducto genital femenino para lograr embarazos. Por ser una técnica de reproducción asistida permite mejorar su tasa de éxito gracia al desarrollo tecnológico en las ciencias médicas y en particular del conocimiento relacionado con la fisiología reproductiva. La misma se recomienda cuando existen alteraciones como: incapacidad para depositar el semen en la vagina, oligo, asteno, terato o azoospermia no aguda, anatomía del cuello uterino no apta para la penetración espermática, endometriosis, disfunción ovolutaria, infertilidad inmunológica o de origen desconocido. Por ello, el momento de la ovulación se convierte en un factor decisivo para determinar su éxito, lo que conduce a establecer una serie de estrategias como: el monitoreo urinario de la hormona LH o la inyección de gonadotropina coriónica humana (HCG) para inducir la ovulación y lograr un mejor control del ciclo. Ante estas acciones, se pueden visualizar los factores de pronósticos que aseguran el éxito del embarazo por inseminación intrauterina; en consecuencia, el especialista considera la edad de la mujer para evaluar la calidad de los ovocitos, número de folículos pre-ovulatorios, grosor endometrial al momento de la ovulación, en cuanto al hombre estima la concentración de espermatozoides móviles poscapacitación con una morfología normal. Además, al incorporar la ecografía vaginal hace posible mantener un estricto control del desarrollo folicular y la correcta sincronización entre la ovulación y el momento de la inseminación. Factores esenciales para estimar el logro del respectivo embarazo intrauterino. Consideraciones de importancia para el desarrollo de este artículo cuyo objetivo es analizar los factores de pronósticos en embarazo tras la inseminación intrauterina, aspecto que permite ubicarlo en el campo de la investigación documental, apoyado en un diseño bibliográfico que da apertura a la construcción de sus conclusiones.

Palabras Claves: Factores de Pronósticos; Embarazo; Inseminación Intrauterina.

\begin{abstract}
Intrauterine insemination is a process characterized by depositing a group of sperm selected and prepared in female genital duct to achieve pregnancies. As a technique of assisted reproduction improve your success rate thanks to the technological development in medical science, and in particular of knowledge related to reproductive physiology. It is recommended when there are alterations such as: failure to deposit the semen in the vagina, oligo, asteno, terato or non-acute azoospermia, not suitable for sperm penetration, endometriosis, dysfunction ovolutaria Anatomy of the cervix, immunological or unexplained
\end{abstract}




\section{Factores de pronósticos en embarazo tras la inseminación intrauterina}

Vol. 3, núm. 3., (2019)

Johnny Wimper Potes Duque; Julio Hugo Procel Aguiño; Henry Cristóbal Yoncón Ramírez; Alexandra Asunción Vanegas Vélez

infertility. Therefore, the time of ovulation becomes a decisive factor for determining its success, which leads to a set of strategies, such as: the monitoring of urinary LH hormone or the injection of human chorionic gonadotropin (HCG) to induce the ovulation and achieve better control of the cycle. Before these actions, you can visualize factors of forecasts that ensure the success of the pregnancy by intrauterine insemination; therefore, the specialist considers the woman's age to evaluate the quality of the oocytes, number of pre-ovulatory follicles, endometrial thickness at the time of ovulation, as the man estimated the concentration of motile sperm post-training with a normal morphology. In addition, by incorporating the vaginal ultrasound it makes it possible to maintain a strict control of follicular development and correct timing between ovulation and the time of insemination. Essential factors to estimate the achievement of the respective intrauterine pregnancy. Considerations of importance to the development of this article which aims to analyze the factors of forecasts in pregnancy after intrauterine insemination, aspect that allows you to locate it in the field of documentary research, supported by a design bibliographic giving opening to the construction of its conclusions.

Key Words: Factors of Forecast; Pregnancy; Intrauterine Insemination.

\section{Introducción.}

Al hacer referencia a la inseminación intrauterina, significa destacar el uso y manejo de técnicas relacionadas con la capacitación del semen, así como la utilización de tratamientos hormonales que favorecen el desarrollo folicular múltiple con el fin de disponer de un mayor número de ovocitos para ser fecundados. Debido a los avances tecnológicos en materia de imagenología se presenta la ecografía vaginal como un medio que le asegura al especialista tratante mantener un estricto control del desarrollo folicular y visualizar el momento oportuno entre la ovulación y la inseminación.

En consecuencia, la práctica médica tiene oportunidad de estimar aquellos parámetros de éxito de la inseminación intrauterina, los mismos se encuentran asociados a la mujer entre ellos: la edad por ser un indicador indirecto de la calidad que presentan los ovocitos, números de folículos preovulatorios, grosor del endometrio en el momento de la ovulación. Los relativos al hombre se citan la concentración de espermatozoides móviles y aquellos relacionados con la pareja se precisa el tiempo de infertilidad 


\section{Factores de pronósticos en embarazo tras la inseminación intrauterina}

Vol. 3, núm. 3., (2019)

Johnny Wimper Potes Duque; Julio Hugo Procel Aguiño; Henry Cristóbal Yoncón Ramírez; Alexandra Asunción Vanegas Vélez

presente entre ellos. Por ello, durante las evaluaciones realizadas, existe un factor esencial que alta significación para lograr la eficiencia de la técnica aplicada, es decir, la ovulación representa un de los factores cruciales para determinar su progresivo desarrollo, situación que conduce a utilizar la gonadotropina Coriónica humana (HCG) para inducir la ovulación.

Cabe agregar que, la mayoría de los embarazos que se consiguen por inseminación intrauterina se producen antes del quinto intento. De hecho, si después de haberse llevado a cabo cuatro ciclos de tratamiento no se ha conseguido la gestación, debe pensarse en un problema a nivel de la fecundación. Es posible que los espermatozoides no lleguen hasta el óvulo por una disfunción en ellos o en las trompas de Falopio. También es posible que sí lleguen pero no fecunden, porque no puedan hacerlo o porque en el ovocito no se encuentren los receptores específicos a los receptores específicos a los espermatozoides. Al respecto, Contreras (2017) define a esta técnica de reproducción asistida "como aquella indicada para parejas y mujeres solteras del mismo sexo, con buen pronóstico reproductivo, pero que tienen posibles problemas para conseguir el embarazo". (p.16)

De acuerdo con lo citado, se puede indicar que cuando las parejas buscan aquellos especialistas en fertilidad, tienen oportunidad de lograr con éxito un embarazo, pues, para la incorporación de la técnica por inseminación intrauterina, se valoran los diferentes factores de pronósticos que le aseguran a las parejas el alcance del respectivo embarazo, dado que, sus condiciones reproductivas al ser diagnosticadas debidamente por el médico, pueden recibir tratamientos hormonales entre otros para asegurar un incremento en la producción de óvulos y mejorar la condiciones de los espermatozoides al referirse a parejas heterosexuales.

En relación con los planteamientos anteriores, se puede resaltar que una vez evaluadas las condiciones de la pareja y el especialista actúa mediante la utilización de la técnica de inseminación 


\section{Factores de pronósticos en embarazo tras la inseminación intrauterina}

Vol. 3, núm. 3., (2019)

Johnny Wimper Potes Duque; Julio Hugo Procel Aguiño; Henry Cristóbal Yoncón Ramírez; Alexandra Asunción Vanegas Vélez

intrauterina, existe una mayor probabilidad de lograr el embarazo, tal como lo asevera Alicante (2016) destaca que:

Existe una tasa acumulada de éxito, en más de la mitad de las pacientes quedan embarazadas cuando se llevan a cabo tres o cuatro ciclos de tratamiento (cerca del $80 \%$ cuando la inseminación intrauterina se realiza con semen de donante). Las posibilidades de gestación gemelar oscilan entre un $15 \%$ y un $20 \%$, siendo los embarazos de trillizos francamente excepcionales. (p.12)

Según los datos referidos por Alicante, se precisa que los factores de pronósticos en el embarazo tras inseminación intrauterina, le asegura a las parejas alcanzar resultados altamente significativos, además, se evidencia la presencia de embarazos múltiples debido a la manipulación que se hace durante la ovulación y la sincronización del proceso de inseminación intrauterina. Es decir, dicho procedimiento es un tratamiento de parejas infértiles afectadas por alteraciones leves en el factor masculino, anovulación, endometriosis mínima y leve infertilidad de causa no determinada. También, sirve para las parejas de mujeres solteras que buscan alcanzar un embarazo.

Por lo tanto, antes de llegar a realizar la inseminación intrauterina, el especialista debe recurrir a evaluaciones caracterizadas por la presencia de estudios dirigidos a el seminograma, recuperación de espermatozoides móviles tras capacitado, hacer una exploración ginecológica completa y ecografía transvaginal, confirma la permeabilidad tubárica, determinar hormonas en la mujer en el tercer día del ciclo y estudiar la serología de la pareja. (RPR, HIV, Hepatitis B y C).

No obstante, las características que determinan al respectivo procedimiento médico, lleva a precisar con anticipación una serie de factores de pronósticos que estiman el éxito o no de los resultados por alcanzar. En esta dirección, Báez (2018) precisa una serie de factores de pronósticos tales como: 


\section{Factores de pronósticos en embarazo tras la inseminación intrauterina}

Vol. 3, núm. 3., (2019)

Johnny Wimper Potes Duque; Julio Hugo Procel Aguiño; Henry Cristóbal Yoncón Ramírez;

Alexandra Asunción Vanegas Vélez

La edad de la mujer menor de 38 años, ovulación multifolicular, estimulación ovárica controlada, adecuada reserva ovárica, ausencia del factor tubárico o endometriosis, ausencia de cirugía pélvica, mejora de la calidad espermática mediante la preparación seminal, obtención de una recuperación de espermatozoides móviles mayor de 6 millones/ml, infertilidad menor de 6 años y realización previa de menos de 4 intentos de inseminación intrauterina. Entre los negativos mayores de 38 años (mujer) reserva ovárica disminuida, presencia de factor tubárica, antecedentes de cirugía pélvica, endometriosis, calidad espermática menor a $6 \times 10_{6} / \mathrm{ml}$, infertilidad mayor de 6 años y número de ciclos de tratamiento mayor de 4 intentos. (p.22)

Los factores citados por este autor, llevan a destacar que para lograr con éxito la inseminación intrauterina, los especialistas al cumplir con aquellos diagnósticos previos como la edad en la mujer, puede proyectar sus beneficios, además, de realizar el conjunto de evaluaciones referidas a sus condiciones orgánicas como la reserva ovárica, factor que presenta importancia para poder llegar a establecer el tiempo básico que se estima en la paciente en cuanto al momento de sincronizar su ovulación con la inseminación intrauterina.

Cada uno de los planteamientos citados en párrafos anteriores, son considerados de importancia, pues, mediantes sus apreciaciones se logra entender que la inseminación intrauterina, para alcanzar resultados viables para las parejas, debe ser realizada mediante una serie de evaluaciones que faciliten al especialista estimar las condiciones fisiológicas de la paciente, en otras palabras, no es sólo la mujer que presenta la infertilidad, pues, el hombre también puede estar en esta relación, lo que conduce a generar entre la parejas dificultades para el respectivo logro del embarazo a través de las relaciones sexuales.

En consecuencia, le corresponde a los especialistas fijar posiciones ante los hallazgos realizados durante el respectivo diagnóstico, se muestra que cuando la mujer es mayor de 38 años, se presentan 


\section{Factores de pronósticos en embarazo tras la inseminación intrauterina}

Vol. 3, núm. 3., (2019)

Johnny Wimper Potes Duque; Julio Hugo Procel Aguiño; Henry Cristóbal Yoncón Ramírez; Alexandra Asunción Vanegas Vélez

pronósticos negativos, además se padece de endometriosis se incrementan la dificultad para alcanzar el éxito, de allí, la importancia de estimar los factores de pronóstico en embarazo tras la inseminación intrauterina. Cabe destacar que cada uno de los diferentes argumentos citados anteriormente, sirven de referencia para la realización del presente artículo, dirigido a lograr una interpretación acerca de los factores de pronósticos en embarazo tras la inseminación intrauterina, razón por la cual, se plantea a continuación los eventos vinculados con la metodología.

\section{Método.}

La sistematización de diferentes argumentos en el desarrollo científico, se encuentran determinados por la presencia de un método encargado de orientar su recorrido científico en relación a un tema en particular; en este caso, al buscar analizar los factores de pronósticos en embarazo tras la inseminación intrauterina, se considera pertinente la aplicación del concepto dado por Valdez (2019) representa "el campo o sendero que a manera de una construcción teórica guía al investigador a conseguir determinados objetivos en su tiempo preciso con actividades que tienen ventajas de disciplinar la acción humana."(p.54)

Por lo tanto, la selección del método no es más que una respuesta escogida para aprender la realidad con todas sus connotaciones y por lo tanto, depende de la creatividad del investigador para utilizar de manera exitosa los mejores procedimientos que le faciliten interpretaciones objetivas de ese contexto previamente seleccionado, es decir, hacer posible una modificación en cuanto a la evaluación de los contenidos que se necesitan para organizar los aspectos que estructuran el desarrollo integral del artículo. En este caso particular, el investigador precisa lo planteado por Valdez (ob.cit) en relación al método crítico "hace posible redimensionar las apreciaciones de otros investigadores para convertirlos en nuevos esquemas que facilitan la construcción del desarrollo del problema al poder concluir o generalizar". (p.57) 


\section{Factores de pronósticos en embarazo tras la inseminación intrauterina}

Vol. 3, núm. 3., (2019)

Johnny Wimper Potes Duque; Julio Hugo Procel Aguiño; Henry Cristóbal Yoncón Ramírez; Alexandra Asunción Vanegas Vélez

En respuesta a las consideraciones dadas en el contexto del método y su incorporación como herramienta básica en todo proceso científico, lleva entonces a ubicar el proceso investigativo en un campo especial del hacer interpretativo. Es decir, se fijan posiciones claras en cuanto al tipo, técnicas documentales y técnicas para la recolección de información que hacen posible el desarrollo del tema seleccionado previamente.

\section{Tipo de Investigación}

El conocimiento científico, puede ser entendido en dos direcciones, la primera como el acto de comprender una realidad que está caracterizada por diferentes elementos propios que al ser valorados logran darle consistencia investigativa y la segunda como un proceso final que hace posible fijar posiciones claras ante los acontecimientos debidamente interpretados, para así configurar sus estructuras metodológicas. En otras palabras, estas acciones llevan al investigador hacia la organización de sus interpretaciones bajo el marco de una tipo de investigación especial.

En consecuencia, el contenido temático fijado para la construcción de este artículo, estima la correspondencia con la investigación documental definida por Arias (2012)

Es un proceso basado en la búsqueda, recuperación, análisis, crítica e interpretación de datos secundarios, es decir, los obtenidos y registrados por otros investigadores en fuentes documentales: impresas, audiovisuales o electrónicas. Como en toda investigación, el propósito de este diseño es el aporte de nuevos conocimientos. (p.27)

Según lo citado por Arias, se precisa que todo proceso investigativo enmarcado en lo documental, busca una interpretación de diferentes criterios de forma crítica, estimado por la selección de aquellos materiales impresos que dan oportunidad de crear nuevas consideraciones generales en cuanto a un tópico debidamente establecido por el investigador para así elaborar nuevos conocimientos científicos. 


\section{Factores de pronósticos en embarazo tras la inseminación intrauterina}

Vol. 3, núm. 3., (2019)

Johnny Wimper Potes Duque; Julio Hugo Procel Aguiño; Henry Cristóbal Yoncón Ramírez; Alexandra Asunción Vanegas Vélez

De este modo, la incorporación de datos especiales, se convierten en una unidad de información que se obtiene durante la ejecución de una investigación. Según su procedencia, los datos se clasifican en primarios, cuando son obtenidos originalmente por el investigador; y secundarios, si son extraídos de la obra de otros investigadores, para así llegar a la selección de las técnicas documentales que dan sustentación al desarrollo de los contenidos previamente organizados para finalmente llegar a formular las respectivas conclusiones vinculadas con el tema previsto en su desarrollo.

\section{Técnicas Documentales}

El contenido temático que ha sido previamente seleccionado, no sólo fija las condiciones metodológicas del artículo, sino que encamina las respectivas ubicaciones de sus aspectos generales; es así como, una vez relacionado su método y tipo de investigación, se comprende la significación que presentan las técnicas documentales, mediante las cuales, el investigador lograr darle la respectiva ubicación a cada uno de los contenidos preestablecidos y así finalmente diseñar el respectivo soporte del material a presentar.

En esta dirección, Arias (ob.cit) define las técnicas documentales como "todas aquellas acciones que suministran información, pueden ser aportadas por documentos, obras, material tecnológico, libros donde se registran informaciones de interés para construir un tema en especial”. (p.32). De allí, que el tema previamente seleccionado para el desarrollo de este artículo, al estar relacionado con los factores de pronósticos en embarazo tras la inseminación intrauterina, lleva al investigar a indagar, revisar, leer, interpretar diferentes documentos referidos al contenido, para luego desglosarlos en apreciaciones generales que dan cabida a nuevas interpretaciones desde una perspectiva innovadora.

\section{Técnicas de Recolección de Información}




\section{Factores de pronósticos en embarazo tras la inseminación intrauterina}

Vol. 3, núm. 3., (2019)

Johnny Wimper Potes Duque; Julio Hugo Procel Aguiño; Henry Cristóbal Yoncón Ramírez; Alexandra Asunción Vanegas Vélez

Dentro del campo científico relativo a la investigación documental, las fuentes encargadas de darles consistencia teórica al estudio de un tema en particular, se encuentran determinadas por la introducción de técnicas que le permiten al investigador proyectar sus apreciaciones hacia otros horizontes. Es así como, Arias (ob.cit) las considera como "medios o materiales, impresas, audiovisuales y electrónicas, que permiten la organización de los contenidos informativos que dan relevancia científica a un tema en general”. (p.36)

Al considerar, el último planteamiento, se puede indicar que para el desarrollo del presente artículo, se asumieron como técnicas de recolección de información, la revisión en los diferentes medos electrónicos, así, como la revisión de libros y revistas que sirvieron para complementar cada uno de los aspectos previamente establecidos en función a la necesidad del tema por redactar y de esta manera, crear nuevos criterios científicos de interés para otros investigadores que manifiesten la necesidad de revisión de sus aspectos generales.

\section{Resultados.}

El desarrollo de los aspectos que estructuran el tema seleccionado para conformar nuevas ideas, se encuentra orientado a presentar una serie de interpretaciones reflexivas vinculadas con los factores de pronósticos en embarazo tras inseminación intrauterina, por lo tanto, su desarrollo se llevará a cabo en una forma global debido al enlace que caracteriza al tema y así ofrece al lector nuevos criterios que dan cabida a una valoración integral de sus argumentos desde perspectivas realmente científicas.

\section{Factores Pronósticos en Embarazo tras Inseminación Intrauterina}

La unión entre un hombre y una mujer trae consigo las relaciones sexuales y por ende el embarazo como representación de dicha actividad sexual. Sin embargo, cuando las parejas no logran mediante las relaciones sexuales espontáneas o coito programado después de doce o veinticuatro meses, 


\section{Factores de pronósticos en embarazo tras la inseminación intrauterina}

Vol. 3, núm. 3., (2019)

Johnny Wimper Potes Duque; Julio Hugo Procel Aguiño; Henry Cristóbal Yoncón Ramírez; Alexandra Asunción Vanegas Vélez

éstos deben recurrir a la asistencia médica como parte básica para garantizarles el desarrollo de un embarazo, es allí, donde los avances tecnológicos en materia de fisiología reproductiva, han dado cabida a la inseminación intrauterina. Tal como lo plantea Barros (2016) la inseminación intrauterina "es una técnica que consiste en el depósito de espermatozoides en el conducto genital femenino sin contacto sexual y con la finalidad de lograr el embarazo”. (p.12)

Cabe agregar que, la inseminación intrauterina ha mejorado su tasa de éxito gracias a los avances tecnológicos y al mejor conocimiento de la fisiología reproductiva. Cuando se habla de inseminación artificial se debe hacer referencia a las técnicas de capacitación del semen, las cuales permiten obtener mayor número de espermatozoides móviles y de morfología normal para depositarse en el interior de la cavidad uterina, así como a la utilización de tratamientos hormonales que ayuden al desarrollo folicular múltiple, con la finalidad de obtener mayor número de ovocitos para ser fecundados.

Por ello, cuando las parejas acuden a la atención del especialista, la inseminación intrauterina se recomienda cuando existen alteraciones como: incapacidad para depositar el semen en la vagina, oligoastenoteratozoospermia no aguda, anatomía no apta del cuello uterino, endometriosis, disfunción ovulatoria, infertilidad inmunológica y de origen desconocido. Está demostrado que la inseminación intrauterina es superior al coito programado en los ciclos naturales o en los ciclos estimulados, la inseminación intrauterina aumenta significativamente las probabilidades de concepción comparada con el coito programado.

Según datos aportados por Barros (ob.cit), se destaca que la:

Tasa de éxito en la inseminación intrauterina varía de centro a centro y depende de diferentes factores. Éstos reportan tasas de embarazo muy bajas (5\%) y muy altas (70\%); sin embargo, del 10 al 20\% de los embarazos clínicos (por ciclos) demuestran límites aceptables para todas las causas. (p. 18) 


\section{Factores de pronósticos en embarazo tras la inseminación intrauterina}

Vol. 3, núm. 3., (2019)

Johnny Wimper Potes Duque; Julio Hugo Procel Aguiño; Henry Cristóbal Yoncón Ramírez; Alexandra Asunción Vanegas Vélez

De acuerdo, con los datos presentados se precisa la significación que presenta la utilización de la inseminación intrauterina por ciclos, pues, las probabilidades se incrementan en la medida que se indica el uso de gonadotropinas para la HOC (asociada con la inseminación intrauterina) se incrementan las probabilidades de embarazo de manera significativa, con razón de probabilidades. El momento de la ovulación es un factor decisivo para determinar el éxito de la inseminación intrauterina; por lo cual se han establecido estrategias, como el monitoreo urinario del pico de LH o la inyección de gonadotropina coriónica humana (hCG) para inducir la ovulación; sin embargo, ninguna de estas técnicas ha demostrado ser superior.

Asimismo, el autor precisa que alrededor del "16\% de las parejas necesitan una inseminación con semen fresco del esposo; es decir, una inseminación intrauterina; representa un volumen pequeño de un preparado con alta concentración de espermatozoides móviles el día de la ovulación" (p.18) Esta técnica, de baja complejidad, ha sido ampliamente utilizada para el tratamiento de parejas con una variedad de diagnósticos; entre ellos, factor cervical, anovulación, factor masculino leve, endometriosis e infertilidad inexplicada. Es de anotar que es un procedimiento simple y poco invasivo, posee una favorable relación costo-efectividad.

Cabe destacar que, el universo cada vez más frecuente de parejas infértiles, el factor masculino constituye un problema de alta prevalencia, describiéndose cifras promedio de $40 \%$ en aquellos que consultan transcurrido más de un año de actividad sexual sin protección. El factor masculino se define como la alteración en dos o más espermiogramas, De cualquiera de los parámetros considerados normales. De esta manera, se considera oligospérmica aquella muestra seminal que posee una concentración espermática inferior a 15 millones $/ \mathrm{mL}$ o un recuento total menor a 39 millones de espermatozoides. Una muestra será astenospérmica si la motilidad progresiva no supera el $32 \%$ y se cataloga de teratospérmica cuando la morfología espermática estricta no alcanza al 4\%. 


\section{Factores de pronósticos en embarazo tras la inseminación intrauterina}

Vol. 3, núm. 3., (2019)

Johnny Wimper Potes Duque; Julio Hugo Procel Aguiño; Henry Cristóbal Yoncón Ramírez; Alexandra Asunción Vanegas Vélez

Una vez confirmado un factor masculino mediante dos espermiogramas, deberá proceder a evaluarse las causas corregibles o modificables que existan en la historia clínica, tales como uso de fármacos que interfieren en la espermatogénesis, ejercicio extremo, aumento de temperatura escrotal, obesidad mórbida, hábitos nocivos como el consumo en exceso de alcohol, tabaco y drogas ilícitas. También es recomendable la evaluación escrotal y testicular mediante eco tomografía Doppler para descartar varicoceles moderados a severos, cuya presencia puede perpetuar el factor masculino y que son corregibles mediante cirugía.

La solicitud de exámenes de laboratorio que permitan reconocer el estado del eje hipotálamohipófisis-testicular es deseable, para lo cual se recomienda evaluar FSH, LH y testosterona total, así como prolactina y hormona estimulante de la tiroides (TSH), dos hormonas que pueden interferir en la pulsatilidad de GnRH y, por ende, afectar dicho eje. Indudablemente, el examen de mayor valor para escoger la técnica de reproducción indicada en casos de factor masculino, es la separación espermática diagnóstica, cuyo resultado, expresada en millones de espermatozoides móviles totales recuperados, será un indicador importante a tener en cuenta.

Por ello, la obligación del especialista en medicina reproductiva es, como en todo el quehacer médico, procurar tratar a las parejas infértiles sin dañar. Esto significa, entre otras cosas, decidir en conjunto con la pareja la modalidad terapéutica más adecuada para ellos, considerando los factores involucrados así como la relación costo/beneficio. La técnica elegida deberá ser proporcional al problema que presentan, con un claro conocimiento de los beneficios versus los riesgos de la misma, así como de las expectativas reales de éxito de dicha terapia según las características de cada pareja. El objetivo es obtener la mayor cantidad de embarazos únicos en el menor número posible de intentos, esto es, la tasa de embarazo único por ciclo, que será nuestro mejor indicador de cómo va con cada técnica en los respectivos centros de medicina reproductiva. 


\section{Factores de pronósticos en embarazo tras la inseminación intrauterina}

Vol. 3, núm. 3., (2019)

Johnny Wimper Potes Duque; Julio Hugo Procel Aguiño; Henry Cristóbal Yoncón Ramírez; Alexandra Asunción Vanegas Vélez

Es impresentable en estos días exhibir tasas altas de embarazo clínico a expensas de multigestación, fenómeno que se observa tanto en baja como en alta complejidad, y que obedece a una vigilancia insuficiente de la respuesta ovárica a la inducción de ovulación, con excesivo número de folículos ovulatorios en el caso de baja complejidad, y de embriones transferidos en alta complejidad. En relación con las causas de la infertilidad femenina como factores pronósticos, es difícil aislar cada una y medir su influencia en el éxito de la inseminación intrauterina.

Varios análisis retrospectivos revelan el efecto negativo de la endometriosis y el factor tubario alterado con el éxito de la inseminación intrauterina. Al igual que en la mujer, es difícil aislar y determinar el efecto de los parámetros masculinos en la tasa de embarazo con la inseminación intrauterina. La falta de unificación de los parámetros normales de la espermatobioscopia es otra de las causas que dificultan la evaluación de sus ventajas en el éxito de la inseminación intrauterina. En la actualidad se considera que la concentración de espermatozoides móviles pos capacitación es el principal pronóstico de éxito. La infertilidad ocasionada por afectación grave del factor masculino requiere técnicas de reproducción asistida de alta complejidad, más que de la inseminación intrauterina. Ésta ha demostrado una función importante en el tratamiento clínico de la subfertilidad masculina. La concentración y movilidad espermática pos capacitación son factores pronósticos muy importantes; sin embargo, se han propuesto otras variables.

\section{Cuadro $N^{\circ} 1$ Tasa de Rango de Tiempo de Infertilidad en Pacientes sometidos a Inseminación}

\section{Intrauterina}




\section{Factores de pronósticos en embarazo tras la inseminación intrauterina}

Vol. 3, núm. 3., (2019)

Johnny Wimper Potes Duque; Julio Hugo Procel Aguiño; Henry Cristóbal Yoncón Ramírez; Alexandra Asunción Vanegas Vélez

\begin{tabular}{|c|c|c|c|c|c|}
\hline \multirow{2}{*}{ Rango } & \multicolumn{2}{|c|}{ Embarazadas } & \multirow{2}{*}{$\begin{array}{c}\text { No. } \\
\text { embarazadas } \\
\text { (n) }\end{array}$} & \multirow{2}{*}{$\begin{array}{c}\text { Tasa } \\
\text { Embarazo } \\
(\%)\end{array}$} & \multirow{2}{*}{$\begin{array}{c}\text { Tasa } \\
\text { acumulada } \\
\text { embarazo } \\
(\%)\end{array}$} \\
\hline & (n) & $(\%)$ & & & \\
\hline $1-3$ & 12 & 19,7 & 32 & 27,3 & 19,7 \\
\hline $4-6$ & 29 & 47,5 & 79 & 26,9 & 67,2 \\
\hline $7-9$ & 14 & 22,9 & 47 & 22,9 & 90,1 \\
\hline $10-12$ & 6 & 9,8 & 60 & 9,1 & 100 \\
\hline $13-15$ & 0 & 0 & 7 & 0,0 & 100 \\
\hline $16-18$ & 0 & 0 & 3 & 0,0 & 100 \\
\hline Más 18 & 0 & 0 & 1 & 0,0 & 100 \\
\hline Total & 61 & 100 & 229 & & \\
\hline
\end{tabular}

Fuente: Barros (ob.cit)

Los hallazgos representados en el cuadro $n^{\circ} 1$, muestran claramente en cuanto al rango de tiempo de infertilidad fue estadísticamente significativo $(\mathrm{p}=0,003)$, entre los grupos. Se encontró que la tasa de embarazo en parejas con menos de cuatro años fue del 19,7 \%, disminuyendo progresivamente hasta hacerse nula en parejas con más de 13 años. Por lo tanto, se puede inferir, que en las parejas cuyo tiempo de infertilidad es menor, existe factores de pronósticos positivos, situación contraria al presentar mayores años de infertilidad.

Los datos representados en el cuadro 2; que se indican a continuación, precisan que la presencia de los diferentes factores de pronósticos la edad, tiempo de fertilidad, densidad espermática y movilidad espermática, constituyen la suma de eventos particulares que tienen mayor incidencia en lograr resultados positivos al momento de alcanzar el respectivo embarazo por inseminación intrauterina, lo que permite deducir que, el especialista durante los procesos de diagnóstico debe considerar como factores esenciales que le darán cabida a una respuesta favorable a la pareja de alcanzar el respectivo embarazo.

Cuadro $N^{\circ} 2$ Tasa de Embarazo en Parejas sometidas a Inseminación Intrauterina de acuerdo con los Diferentes Factores Pronósticos 


\section{Factores de pronósticos en embarazo tras la inseminación intrauterina}

Vol. 3, núm. 3., (2019) Johnny Wimper Potes Duque; Julio Hugo Procel Aguiño; Henry Cristóbal Yoncón Ramírez; Alexandra Asunción Vanegas Vélez

\begin{tabular}{|l|c|c|c|}
\hline \multicolumn{1}{|c|}{ Variables } & Embarazadas $^{* *}$ & No embarazadas & P \\
\hline Edad (años) & $33,3 \pm 3,7$ & $33,6 \pm 3,4$ & 0,431 \\
\hline $\begin{array}{l}\text { Tiempo de infertilidad } \\
\text { (años) }\end{array}$ & $5,7 \pm 2.5$ & $6,8 \pm 3,4$ & $0,005^{*}$ \\
\hline $\begin{array}{l}\text { Densidad espermática } \\
\text { (millones/ml) }\end{array}$ & $76,8 \pm 31,3$ & $73,7 \pm 33,3$ & 0,425 \\
\hline $\begin{array}{l}\text { Movilidad espermática } \\
\text { (\%) }\end{array}$ & $77,9 \pm 11,5$ & $73,7 \pm 15,5$ & $0,015^{*}$ \\
\hline No. de folículos maduros & $3,2 \pm 2,2$ & $2,7 \pm 2,2$ & 0,832 \\
\hline No. de folículos basales & $10,5 \pm 4,8$ & $9,8 \pm 5,2$ & 0,794 \\
\hline Grosor Endometrial & $10,2 \pm 1,9$ & $10,9 \pm 2,3$ & 0,326 \\
\hline Día de Inseminación & $12,9 \pm 1,2$ & $13,2 \pm 1,3$ & 0,223 \\
\hline Número de ciclos & $2,3 \pm 0,9$ & $2,6 \pm 1,1$ & $0,029^{*}$ \\
\hline
\end{tabular}

* Variable con significación estadística $(p<0,05)$.

** Valores expresados en medias \pm DE.

Fuente: Barros (ob.cit)

Otro factor de pronóstico en embarazo tras inseminación intrauterina de importancia para los especialistas lo representa la edad de la mujer encontrando que aquellas que tienen menos de 30 años, tienen una posibilidad representada del $24,4 \%$, asimismo, las que se ubican en un rango entre 30 y 34 años respectivamente su tendencia es de $24,1 \%$; no obstante, en la medida que se incrementa la edad se visualiza un descenso significativo en la posibilidad de un embarazo como lo muestra el rango entre 35 a 39 años respectivamente. Datos que son representados a continuación en el cuadro n ${ }^{\circ} 3$.

\section{Cuadro $N^{\circ} 3$ Tasa de Embarazo según la Edad de la Paciente}

\begin{tabular}{l|c|c|c|c|}
\hline \multicolumn{1}{c|}{ Edad } & $\begin{array}{c}\text { Embaraz } \\
\text { adas }(\mathrm{n})\end{array}$ & $\begin{array}{c}\text { Embarazadas } \\
\text { Em) }\end{array}$ & $\begin{array}{c}\text { Embarazadas } \\
(\%)\end{array}$ & $\begin{array}{c}\text { Tasa } \\
\text { Embarazo } \\
(\%)\end{array}$ \\
\hline$<30$ años & 11 & 34 & 18,0 & 24,4 \\
\hline 30 a 34 años & 26 & 82 & 42,6 & 24,1 \\
\hline 35 a 39 años & 17 & 86 & 27,9 & 16,5 \\
\hline 40 años y más & 7 & 27 & 11,5 & 20,6 \\
\hline Total & 61 & 229 & 100 & 21,0 \\
\hline
\end{tabular}




\section{Factores de pronósticos en embarazo tras la inseminación intrauterina}

Vol. 3, núm. 3., (2019)

Johnny Wimper Potes Duque; Julio Hugo Procel Aguiño; Henry Cristóbal Yoncón Ramírez; Alexandra Asunción Vanegas Vélez

Fuente: Barros (ob.cit)

Es importante acotar que, después de la inseminación intrauterina, la paciente recibe un suplemento hormonal con el fin de mejorar las condiciones del endometrio para la implantación embrionaria. No se aconseja ninguna restricción, ni en la dieta, ni en la actividad física de ningún tipo. El reposo no va a mejorar el pronóstico de embarazo. La paciente puede notar ligeras molestias o incluso un ligerísimo marcado después de la inseminación, que es considerado normal. Dos semanas después, se programa la realización de una prueba de embarazo. Antes, es posible que la paciente no note absolutamente nada o quizá una moderada tensión mamaria, por la progesterona, e incluso en ocasiones, molestias similares a las de la menstruación. Ninguno de estos síntomas son indicativos de éxito o de fracaso y solo se sabe con certeza si se ha producido la gestación cuando se obtenga el resultado de la prueba de embarazo. Los resultados con este tratamiento son de, aproximadamente, un $20 \%$ de gestaciones por ciclo de inseminación artificial. Es decir, aproximadamente una de cada cinco mujeres queda embarazada en el primer intento.

En consecuencia, al hacer referencia a la inseminación intrauterina, se precisa, es un procedimiento para tratar la infertilidad. La misma se realiza con el esperma que se lavó y concentró se coloca directamente en el útero cerca de la fecha en la que el ovario libera uno o más óvulos para que sean fertilizados. En los tipos de inseminación intrauterina más antiguos, el esperma se colocaba en la vagina. Si bien esto era más fácil, no tenían tanto éxito como el procedimiento actual. El resultado que se espera de la inseminación intrauterina es que el esperma llegue a la trompa de Falopio y fertilice un óvulo que está esperando, lo que produce un embarazo normal. Según los motivos de la infertilidad, la inseminación intrauterina se puede coordinar con el ciclo normal o con los medicamentos para la fertilidad. 


\section{Factores de pronósticos en embarazo tras la inseminación intrauterina}

Vol. 3, núm. 3., (2019)

Johnny Wimper Potes Duque; Julio Hugo Procel Aguiño; Henry Cristóbal Yoncón Ramírez; Alexandra Asunción Vanegas Vélez

Cabe agregar que, la capacidad de una pareja para quedar embarazada depende de muchos factores diferentes. Por ello, la inseminación intrauterina se utiliza con mayor frecuencia en parejas que tienen, según Jaramillo (2016) una serie de factores entre los cuales destaca los siguientes:

Esperma de un donante. Para las mujeres que necesitan usar esperma de un donante para quedar embarazadas, la inseminación intrauterina es el procedimiento más frecuentemente utilizado para lograr el embarazo. Los especímenes congelados de esperma de un donante se obtienen de laboratorios certificados y se descongelan antes del procedimiento de la inseminación intrauterina.

Infertilidad sin causa aparente. La inseminación intrauterina se realiza a menudo como un primer tratamiento para la infertilidad sin causa aparente junto con medicamentos inductores de la ovulación.

Infertilidad relacionada con la endometriosis. Para la infertilidad relacionada con la endometriosis, el uso de medicamentos para obtener un óvulo de buena calidad junto con la realización de la inseminación intrauterina es a menudo el primer enfoque del tratamiento.

Infertilidad de factor masculino leve (subfertilidad). El análisis del semen de la pareja, es uno de los primeros pasos en la evaluación médica de la infertilidad, puede mostrar una concentración de esperma inferior al promedio, un movimiento débil (motilidad) de los espermatozoides o anomalías en el tamaño y la forma del espermatozoide (morfología). La inseminación intrauterina puede superar algunos de estos problemas, pues, la preparación del esperma para el procedimiento ayuda a separar los espermatozoides altamente móviles, normales de los de menor calidad.

Infertilidad del factor cervical. El cuello uterino, en el extremo inferior del útero, proporciona la abertura entre la vagina y el útero. La mucosidad producida por el cuello uterino alrededor del momento de la ovulación brinda un ambiente ideal para que los espermatozoides se desplacen desde la vagina hasta 


\section{Factores de pronósticos en embarazo tras la inseminación intrauterina}

Vol. 3, núm. 3., (2019)

Johnny Wimper Potes Duque; Julio Hugo Procel Aguiño; Henry Cristóbal Yoncón Ramírez; Alexandra Asunción Vanegas Vélez

las trompas de Falopio. Pero, si el moco cervical es demasiado espeso, puede impedir el recorrido del esperma. La inseminación intrauterina evita el cuello del útero, depositando el esperma directamente en el útero y aumentando el número de espermatozoides disponibles para encontrarse con el óvulo a la espera.

Alergia al semen. Rara vez, una mujer podría tener una alergia a las proteínas en el semen de su pareja. La eyaculación en la vagina causa enrojecimiento, ardor e hinchazón cuando el semen entra en contacto con la piel. Un preservativo puede protegerte de los síntomas, pero también previene el embarazo. Si la sensibilidad es severa, la inseminación intrauterina puede ser eficaz, ya que muchas de las proteínas del semen se eliminan antes de introducir el esperma.

De este modo, se entiende que para el desarrollo de dicho procedimiento implica una coordinación minuciosa antes del procedimiento en sí, en esta dirección Jaramillo (ob.cit) enumera los diferentes pasos a seguir:

Preparar la muestra de espermatozoide: La pareja proporciona una muestra de espermatozoides en el consultorio del médico o se puede descongelar un vial de espermatozoides congelados de un donante y prepararse. Debido a que los elementos presentes en el semen, que no son espermatozoides, pueden causar reacciones en el cuerpo de la mujer que interfieren con la fertilización, la muestra se lavará de tal manera que se separe a los espermatozoides normales y altamente activos de los de más baja calidad y de otros elementos. La posibilidad de quedar embarazada aumenta mediante el uso de pequeñas muestras de espermatozoides sanos altamente concentradas.

Control de la ovulación. Debido a que el tiempo de la inseminación intrauterina es fundamental, resulta esencial controlar los signos de la ovulación inminente. Para hacer esto, es posible que uses un kit de predicción de la ovulación mediante el análisis de orina para realizarse en el hogar, que detecta el momento en que el cuerpo produce un aumento o una liberación de la hormona luteinizante. O, puede realizarse un método de pruebas por imágenes que le permita al médico visualizar los ovarios y el 


\section{Factores de pronósticos en embarazo tras la inseminación intrauterina}

Vol. 3, núm. 3., (2019)

Johnny Wimper Potes Duque; Julio Hugo Procel Aguiño; Henry Cristóbal Yoncón Ramírez; Alexandra Asunción Vanegas Vélez

crecimiento de los óvulos (ecografía transvaginal). También es posible que te administren una inyección de coriogonadotropina humana para que ovules uno o más óvulos en el momento correcto.

Determinar el momento perfecto. La mayoría de las inseminaciones intrauterinasse realiza uno o dos días luego de detectar la ovulación. Tu médico debe tener un plan detallado para el momento de la inseminación intrauterina.

La consulta para la inseminación intrauterina toma alrededor de 15 a 20 minutos y, por lo general, se realiza en la clínica o el consultorio del médico. El procedimiento de la inseminación intrauterina en sí toma solo un minuto o dos, y no requiere de ningún medicamento ni analgésico. El procedimiento lo realiza el médico especializado. Mientras estás recostada en una camilla de exploración, debe colocar las piernas en los estribos y se colocará un espéculo en la vagina, similar a lo que experimentas durante un Papanicolaou. Durante el procedimiento, el médico conecta un vial que contiene una muestra de espermatozoides saludables al extremo de un tubo largo, delgado y flexible (catéter). Coloca el catéter en la vagina, a través de la apertura del cuello uterino y hasta el útero. Impulsa la muestra de espermatozoides a través del tubo hasta el útero. Retira el catéter y luego el espéculo.

Por lo tanto, los factores que aumentan las posibilidades de éxito de embarazo con técnica de reproducción asistida en la mujer suele ser predominantemente la edad, de forma que a partir de los 35 años la probabilidad de embarazo disminuye hasta el 66\%. Otros factores que influyen en el éxito de embarazo es la causa de la esterilidad de la mujer, el estado de las trompas uterinas.

Figura $N^{\circ} 1$ Inseminación Intrauterina 


\section{Factores de pronósticos en embarazo tras la inseminación intrauterina}

Vol. 3, núm. 3., (2019)

Johnny Wimper Potes Duque; Julio Hugo Procel Aguiño; Henry Cristóbal Yoncón Ramírez; Alexandra Asunción Vanegas Vélez

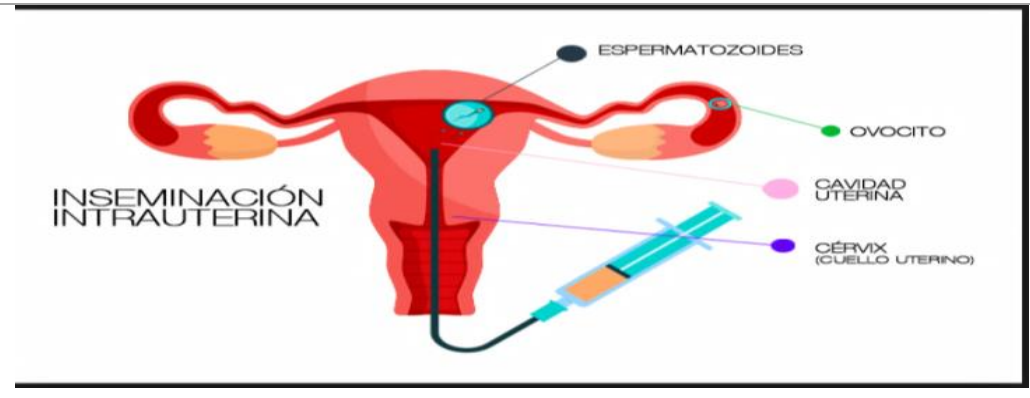

Fuente: Elaboración Propia (2019)

De los planteamientos anteriores, se destaca que la inseminación intrauterina, presenta una gama de factores de pronósticos en embarazo, donde la parte masculina se le atribuye otros factores que intervienen en las posibilidades de éxito de embarazo. Entre ellos cabe destacar, al igual que en la mujer, la edad del varón. A partir de los 40 años su fertilidad declina en un 70\%. A medida que el varón envejece, el ADN presente en su esperma comienza a fragmentarse, es decir, se provocan roturas o lesiones en el material genético del espermatozoide, lo que provoca infertilidad. Así mismo, otro elemento clave es la calidad del semen, existiendo 5 veces más posibilidades de gestación en el caso de hacer una inseminación artificial.

No obstante, independientemente de los factores del hombre y la mujer que influyen directamente en la tasa de éxito del embarazo, cabe considerar las causas de éxito relacionadas con el propio tratamiento. De esta forma, habría que tener en cuenta el número de folículos desarrollados como respuesta al tratamiento y la cantidad de espermatozoides útiles obtenidos a partir de las muestras seminales. Se puede decir que en los grupos de peor pronóstico está en torno al $20 \%$ de éxito de embarazo, mientras que puede aproximarse al 50\% en los pacientes con perspectivas más favorables.

En consecuencia, para controlar los factores de pronósticos en embarazo tras inseminación intrauterina, la preparación de la muestra de semen, además de eliminar algunos componentes, busca mejorar la concentración de espermatozoides y la selección de aquellos que presenten buena movilidad, 


\section{Factores de pronósticos en embarazo tras la inseminación intrauterina}

Vol. 3, núm. 3., (2019)

Johnny Wimper Potes Duque; Julio Hugo Procel Aguiño; Henry Cristóbal Yoncón Ramírez; Alexandra Asunción Vanegas Vélez

de los diferentes métodos de preparación del semen para separar los espermatozoides móviles, morfológicamente normales del plasma seminal y eliminar generalmente prostaglandinas, leucocitos, bacterias y espermatozoides inmóviles; tienen resultados aceptables.

Estos planteamientos, llevan a resaltar que mediante la inseminación intrauterina es la primera opción de tratamiento de la pareja infértil, en pacientes con infertilidad inexplicada, endometriosis mínima y leve, subfertilidad por factor cervical o subfertilidad por factor masculino leve. Pero su efectividad ha sido cuestionada por sus bajas tasas de éxito y los probables riesgos como el embarazo múltiple y el síndrome de hiperestimulación ovárica. A pesar de ello, sigue siendo una técnica ampliamente usada por su simplicidad ofrece ventajas, como obtener mayores tasas de éxito y la posibilidad de transferencia de embrión único y transferencia posterior de embriones congelados, con lo cual disminuyen los riesgos, a pesar de ser mucho más costosa.

De lo antes expuesto, se precisa que mediante la utilización de la inseminación intrauterina, los especialista en fertilidad pueden estimar con anticipación los factores de pronósticos para el logro de un embarazo una vez realizada la respectiva técnica; sin embargo a pesar de que las tasas de éxito no son muy altas, es ampliamente usada cuando no existe obstrucción tubárica bilateral o infertilidad masculina severa. Quienes más se benefician son las mujeres jóvenes, con trompas permeables, infertilidad menor a tres años, sin trastorno ovulatorio, sin endometriosis moderada o severa y sin un factor masculino severo; por lo tanto, la selección adecuada de las pacientes es importante para obtener mejores resultados.

\section{Conclusiones.}

El desarrollo de los diferentes argumentos presentados a lo largo de la construcción del presente artículo cuyo objetivo fue analizar los factores pronósticos en embarazo tras inseminación intrauterina, se puede concluir lo siguiente: 


\section{Factores de pronósticos en embarazo tras la inseminación intrauterina}

Vol. 3, núm. 3., (2019)

Johnny Wimper Potes Duque; Julio Hugo Procel Aguiño; Henry Cristóbal Yoncón Ramírez; Alexandra Asunción Vanegas Vélez

El proceso de inseminación intrauterina, por ser una técnica que consiste en depositar una muestra

de semen en el tracto reproductor femenino (vagina). Previamente para potenciar la capacidad de fertilización espermática, se realizan una serie de mejoras en el laboratorio con objeto de concentrar y vitalizar el semen. Es requisito necesario que la mujer tenga al menos una trompa uterina permeable.

En consecuencia, se puede indicar que entre los factores pronósticos de éxito en una inseminación intrauterina varían según diferentes reportes, por lo que no se pueden dar con bases más objetivas. Esto es el reflejo de tratar pacientes con variabilidad biológica marcada en su reacción al tratamiento. Entre los más significativos se precisa la edad de la mujer se relaciona directamente con la fecundidad, pues disminuye con el paso de los años debido entre otras cosas a una reducción de la receptividad uterina y una disminución en la calidad de los ovocitos. En este estudio, la tasa de éxito es nula en las pacientes mayores de 39 años.

El mejor tratamiento es el que se basa en las características individuales de cada paciente tales como edad, tiempo de evolución de la esterilidad, tipo de esterilidad, hijos anteriores, pues hay características que se asocian con una mayor probabilidad para lograr gestación evolutiva tanto de forma espontánea como con ciclos de inseminación artificial: mujer joven, esterilidad secundaria, menor tiempo de duración de la esterilidad.

Por ello, la fertilidad está influida por muchos factores: la edad de la mujer, reserva ovárica, calidad del esperma. Por eso, lo primero que se debe hacer es acudir al médico para que estudie las causas que dificultan la fecundación, y cuáles son tus posibilidades de conseguir un embarazo. Las probabilidades de éxito son más altas en aquellas mujeres o parejas con problemas leves para conseguir el embarazo: Hombres con pocos espermatozoides o con problemas de impotencia. Mujeres con problemas de ovulación. Mujeres con miomas o endometriosis leve. Otros factores de buen pronóstico, ya que están no relacionados con problemas de fertilidad, son: Mujeres sin pareja. Mujeres homosexuales. 


\section{Factores de pronósticos en embarazo tras la inseminación intrauterina}

Vol. 3, núm. 3., (2019)

Johnny Wimper Potes Duque; Julio Hugo Procel Aguiño; Henry Cristóbal Yoncón Ramírez; Alexandra Asunción Vanegas Vélez

En los dos últimos casos, se recurre a esta técnica porque se requiere un donante y no porque existan problemas de fertilidad. Por este motivo, estas mujeres suelen conseguir el embarazo en pocos ciclos. Otro de los consejos para una inseminación intrauterina exitosa, es el seguimiento de la ovulación. Es primordial que la inseminación tenga lugar en el momento óptimo del ciclo, es decir, durante los días fértiles. El equipo de profesionales realizará un seguimiento para identificar cuando es el mejor momento para que se produzca la fecundación.

\section{Bibliografía.}

Alicante, G. (2016). Tasa de Fertilidad. IMBIOMED, 12.

Arias, F. (2012). El Proyecto de Investigación: Introducción a la Metodología Científica. Caracas: Espinteme.

Báez, G. (2018). Guía Completa de Reproducción Asistida. ARS, 22.

Barros, J. (2016). Factores Pronósticos en Embarazo por Inseminación Intrauterina. Medigraphic, 12.

Contreras, M. (2017). Embarazo en Pacientes con Infertilidad Primaria. Médica, 16.

Jaramillo, P. (2016). Inseminación Intrauterina e In Vitro. España: Gedisa.

Valdez, J. (2019). Métodos y Procedimientos Metodológicos. México: Limusa.

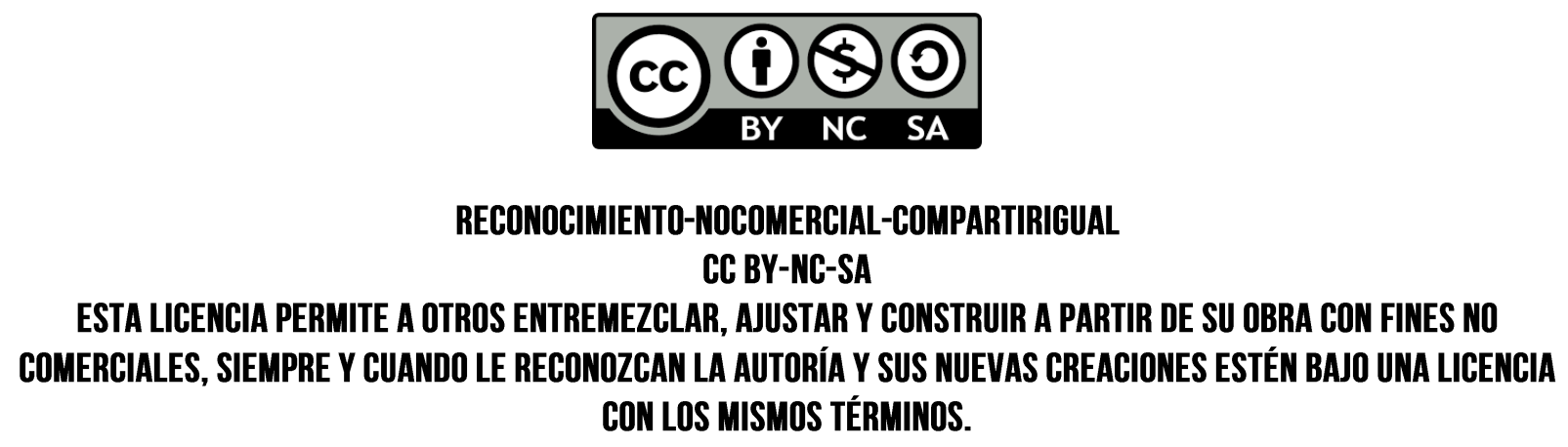

